DOI: $\underline{\text { https://doi.org/10.24297/jap.v17i.8623 }}$

\title{
Integration of Life and Consciousness into Cosmology
}

\author{
Amrit Šorli Srecko*, Štefan Čelan \\ Bijective Physics Institute*, Slovenia, Scientific Research Centre Bistra, Slovenia \\ sorli.bijective.physics@gmail.com, stefan.celan@bistra.si \\ https://orcid.org/0000-0001-6711-4844*
}

\begin{abstract}
The Big Bang model is based on vague interpretations of experimental data. Direct interpretation of the data opens a new vision of the universe in a permanent dynamic equilibrium without beginning and without end. In the universe as the main system, the evolution of life on planet Earth is a consistent part of the universal process that operates in the entire universe. The origin of life as a consistent part of universal dynamics is in higher dimensions of the multidimensional dynamic quantum vacuum.
\end{abstract}

Keywords: multidimensional dynamic quantum vacuum, the origin of life, $\mathrm{CMB}$, cosmological redshift

\section{Introduction}

Cosmology, in order to produce meaningful results, has to start with the shape of the universal space. NASA's result, confirmed by their and others' measurements have proved universal space has Euclidean shape with only a $0.4 \%$ margin of error [1]. This means that the correct model in cosmology is Euclidean space. We cannot use Riemann's finite geometry in the description of the universal space. NASA's results confirm that universal space is infinite, which means the amount of energy in the universe is infinite. The universe cannot be approached as a finite system; the universe is an infinite system.

We can define the position of an object $A$ in space with respect to object $B$ with three coordinates. This does not mean, however, that universal space is three-dimensional. About the dimensionality of the universal space, we do not yet know and may never know. As mathematics allows that Euclidean space has more than three dimensions, we cannot exclude that universal space is multidimensional too. Research by Bronnikov and others suggests that the universe has inhomogeneous compact extra dimensions [2]. This article develops the idea that these extra dimensions have a physical basis in the dynamic vacuum, which is multidimensional. Gravity is the result of vacuum fluctuations in the $4^{\text {th }}$ dimension of the multidimensional dynamic quantum vacuum (vacuum). A three-dimensional physical object reduces the density of the $4^{\text {th }}$ dimension of the dynamic vacuum equal to the amount of its energy:

$\frac{E}{c^{2}}=m=\left(p_{\max }-p_{\min }\right) \cdot V(1)$,

where $\rho_{\max }$ is the density of the vacuum in interstellar space, $\rho_{\min }$ is the density of the vacuum on the surface of the physical object and $V$ is the volume of the object [3]. Vacuum fluctuations that flow from $\rho_{\max }$ towards $\rho_{\min }$ are the origin of gravity, which is the main force of universal dynamics.

\section{The Universe is a system in a permanent dynamic equilibrium}

The dynamic quantum vacuum model replaces space-time as the fundamental arena of the universe $[3,4]$. In the vacuum, the time has merely a mathematical existence, namely, time is the numerical sequential order of events running in the vacuum [5]. The vacuum is timeless in the sense that time is not its $4^{\text {th }}$ dimension. The vacuum is 
the direct information medium of entanglement regarding EPR-type experiments [6]. In the universe, past and future have only a mathematical existence. Irreversible changes are running in the timeless space that originates in the vacuum. The term "physical past" has no corresponding physical reality.

We do not have any experimental data that confirm that the cosmic microwave background radiation (CMB) exists in some fundamental physical arena called "space-time". CMB is measured only in universal space, not in time. Time is only the mathematical parameter of changes running in space. In this sense, universal space is time-invariant and has no physical past. The cosmological principle also is time-invariant [4]. Events in the universe run in timeless space and are irreversible. We experience events that have happened in timeless space as "past". As the cosmological principle is time-invariant (means that potential observer in any possible point of the universe would have the same view on the universe independently on time) the possibility of the "recombination period" ever has happened is excluded. The universe as we see it today was (in the sense of past events that has happened in timeless space) and will (in the sense of coming events) remain the same from eternity to eternity: a vast infinite space with numerous galaxies where the old matter is transformed into fresh energy in the form of elementary particles. Black holes are rejuvenating systems of the universe[4]. We observe $\mathrm{CMB}$ in the last few decades, CMB is not proving that the "recombination period" has happened, it is only showing the universal space radiates CMB. Thinking, that CMB is proving the Big Bang cosmology model is a fanciful idea, it is not scientific reasoning.

Cosmological redshift data also do not prove the expansion of the universe. This is an incorrect interpretation. When light from distant galaxies reaches the Earth, its frequency decreases. On the path to the Earth, light loses some of its energy because it is moving against the gravitational flow of the vacuum, which is pointing in the direction of galaxies:

$E_{\text {photonEarth }}=E_{\text {photongalaxy }}-\Delta E(2)$,

where $E_{\text {photongalaxy }}$ is the energy of the photon in the galaxy, $E_{\text {photonEarth }}$ is the energy of the photon arriving on the Earth and $\Delta E$ is the loss of energy due to the gravitational flow of the vacuum.

$\Delta E=h \cdot \Delta v(3)$,

where $h$ is Planck's constant and $\Delta v$ is the reduction of photon frequency due to gravitational vacuum fluctuations.

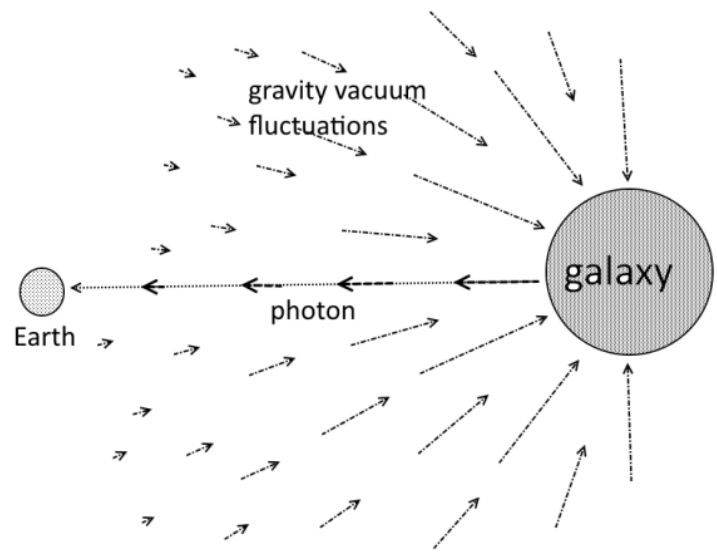

Figure 1: Red-shift caused by vacuum fluctuations

The Swiss astronomer Zwicky named this phenomenon "tired light" [7]. Recent research confirms that the idea of an accelerating universe is not in accord with astronomical observations: "Using the largest available supernova data set, the JLA catalogue, we find that the timescape model fits the luminosity distance-redshift 
data with a likelihood that is statistically indistinguishable from the standard spatially flat $\Lambda$ cold dark matter cosmology by Bayesian comparison. In the timescape case, cosmic acceleration is non-zero but has a marginal amplitude, with best-fitting apparent deceleration parameter, q0 $=-0.043+0.004-0.000$ " [8].

Several data obtained in astronomy at the end of the last century have shown that the observable universe is $10^{4}$ times larger than the universe according to the Big-Bang model if it expanded at the velocity of light [9]. Advocates of the Big Bang model argue that this discrepancy is due to the curvature of universal space. This argument fails because, as previously described, NASA has measured universal space, showing a Euclidean geometry. It is "flat" [1]. Thus, the Big Bang model is built on a misunderstanding of time as some physical reality in which the universe exists and an incorrect interpretation of obtained data for the CMB and redshift of the light from distant galaxies.

The Big Bang model has a beginning and therefore, somehow needs a "creator". Thinking of the universe as something that could have a beginning in some physical past (which, as shown in this article, is non-existent) is not appropriate. The Big Bang model belongs to the history of physics. The universe is a non-created system in a permanent dynamic equilibrium. On the surface of black holes, the vacuum density is so low that atoms become unstable [3]; they are "old" mater" which transforms back into the "fresh" energy in the form of elementary particles and cosmic rays. Black holes are emanating elementary particles and cosmic rays in interstellar space where they are the "stuff" for the creations of new stars. Black holes are rejuvenating systems of the universe. Almost all galaxies have supermassive black holes in their centre [10]. The energy circulation "black holes $\rightarrow$ elementary particles, cosmic rays $\rightarrow$ formation of stellar objects $\rightarrow$ the disintegration of matter on the surface of the black holes" is continuous. It has no beginning and will have no end.

The idea of the universe having a beginning is the result of a model in which the universe exists in physical time. In order to progress cosmology, we must stop projecting linear time, which originates in the human mind, onto the universe. We are experiencing the flow of changes running in universal space in the frame of psychological time, which exists only in the mind. In the universe, time is merely a mathematical existence; time is the numerical sequential order of changes running in space, which is timeless in the sense that time is not its $4^{\text {th }}$ dimension [9].

Big Bang model suggests that the universe started with a huge explosion that causes the expansion of the universal space. A few seconds after the explosion the size of the universe was relatively small in comparison to today's mapped universe. NASA results which are the most accurate obtained data suggest that the universe has Euclidean shape [1], which means it is infinite. The amount of universal space energy and also energy in the form of matter in the universe is infinite. We know in physics that energy cannot be created and destroyed, it can only transform into another type of energy. The Big Bang model is not in accord with the first law of thermodynamics. On the question: "How finite energy at the beginning has turned into the finite energy of today's universe?", the Big Bang model has no answer [4].

\section{Higher Dimensions of the Vacuum in the living Organism}

The function of the dynamic vacuum as the direct information medium in EPR type experiments [6] can also be extended to living matter, meaning living organisms. Here, there is constant communication between cells via coherent electromagnetic fields, which can be seen according to quantum electrodynamics (QED) as the formations of the vacuum itself [11]. This experimental data opens new perspectives in biology, where a living organism is seen as a living physical object in which the vacuum, plays an important communication role in intercellular communication. The idea of the relatedness of life with the vacuum was also studied by Grandpierre Attila [12] and Igor Jerman [13]. From the perspective of their research, the dynamic vacuum governs the active behavior of living organisms via the electromagnetic field. In a living organism, we have electromagnetic fields that are interrelated with its atomic and molecular structures.

Higher-dimensional vacuum fluctuations in a living organism are stronger than in the same dead organism. We can express this energy difference between a living and the same dead organism as follows: 
$E_{\text {life }}=E_{\text {dead }}+E_{v}(4)$,

where $E_{\text {life }}$ is the energy of the living organism, $E_{\text {dead }}$ is the energy of the dead organism, and $E_{v}$ are higher-dimensional vacuum fluctuations characteristic of the living organism. Slawinski has measured that vacuum fluctuations in the form of electromagnetism vanish at the time of death: biophoton emission at the time of death is about 10 to 100 times stronger than biophoton emission of the living organism [14]. Biophoton emission cannot influence the mass of the dead organism because the emission of the biophotons does not change the nuclei of the atoms that build a living organism. The mass of a given system decreases when nuclei are falling apart. This happens for example in an atom bomb and a huge amount of electromagnetic energy in the form of heat and light is released. Despite biophoton emission, we can write the following formula:

$m_{\text {life }}=m_{\text {dead }}(5)$,

where $m_{\text {life }}$ is the mass of the living organism, $m_{\text {dead }}$ is the mass of the dead organism.

Equation (5), which denotes equality of living mass and dead mass, is in accord with today's physics. However, experimental data obtained back in 1987-90 at the University of Ljubljana shows that gravity is stronger on the living organism as on the same dead organism.

\section{Material and Methods}

Using earthworms (Lumbricus terrestris), the measured difference in gravity on the living and the same dead organism is about one million part of living mass. Five grams of earthworms, at the time of death, lost about five micrograms. 70 grams of the earthworms lost about 70 micrograms [15], which we express with the formula below:

$F_{g(\text { life })}=F_{g(\text { dead })}+\Delta F_{g}(6)$

The experiment was repeated twelve times. Statistical analysis of the obtained data confirms that data are correct within 95\%. In order to exclude human factors, the experiment was repeated six times in September 2018 on a highly precise Mettler Toledo mass comparator AX107H where data are directly transferred from the mass comparator to the computer without human reading.

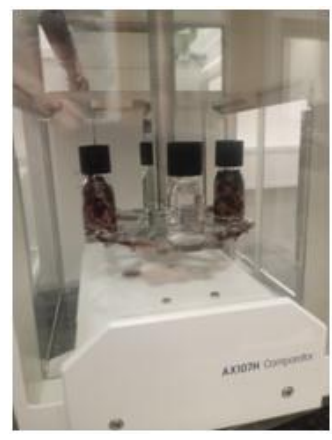

Mettler-Toledo mass comparator AX107H with 2 test tubes with distilled water and two test tubes with worms.

The same results as back in 1987-90 were obtained. The weight of the five grams of living worms diminishes 15 minutes after death by five micrograms. The weight difference remains the same for the next two hours. The average loss of weight by the earthworms is one million part of their living weight. 
With today's mass measuring technology this result is easy to verify; the human factor of the experiment is zero. The results of the measurement are transferred from the balance directly to the computer. We encourage biologists who are interested in this experiment to carry out their independent repetition. This experiment needs a few independent repetitions in order to become recognized by the international scientific community. Repeating the experiment with worms is a necessary killing of living organisms in order to prove the existence of the higher dimensional layers of the vacuum on the gravity. The author does not encourage researchers to repeat this experiment with higher organisms. It would be suitable to prolong the experiment on 24 hours in order to see if perhaps the weight difference will increase. We cannot exclude that the separation between molecular structure and higher dimensional levels of the vacuum takes more than two hours. It might be longer, and the weight difference will increase.

The diminished gravitational force on dead organisms cannot be explained in the frame of Einstein's "massenergy equivalence", namely, that biophoton emission at death could cause such a reduction of energy, which would result in a reduction of gravity for $\Delta F_{g}$. When ten micrograms of the matter are transformed into electromagnetic energy (according to the formula $m=E / c^{2}$ ), a huge release of energy is expected. There is no such release of energy at the time of death. The "gravity gap" $\Delta F_{g}$ between living and the same dead organism in this article is interpreted as a result of higher-dimensional vacuum fluctuations in the living organism that are characteristic of living organisms. The weight of the living organism has two components. The first component is its mass. The second component is the higher-dimensional vacuum fluctuations, which are characteristic of a living organism. Their presence in the living organism increases its weight.

The experiment where the difference between a living and dead organism's weight was measured was first done by American physician Duncan MacDougall in 1907. He was measuring dying people and got the result of around 21 grams of loss of weight at the time of death. The New York Times published an article about his results [16]. At that time there was no theoretical background for these results and science has ignored Duncan MacDougall's research. It has taken me 30 years to develop the theoretical model of higher dimensional layers of the vacuum, which explains the difference in weight between living and dead organisms.

\section{Higher-dimensional vacuum fluctuations and "Orch OR" theory}

The experiment with worms shows that living organisms there is an unknown type of energy which increases the weight of a living organism. We suggest that higher-dimensional quantum fluctuations that are characteristic of living organisms increase their weight. These higher dimensional quantum fluctuations are existing in entire universal space, they represent a physical circumstance for the development of life; they are carrying the information of life development. Based on the experiment with the worms, we are proposing the existence of structures of the universe that are of the essential importance in the development of life.

This our hypothesis is in agreement with orchestrated objective reduction theory (Orch OR), which sees life and consciousness as phenomena that are deeply related to the structures of the universe: "The DP (Diósi-Penrose) form of $O R$ is related to the fundamentals of quantum mechanics and space-time geometry, so Orch OR suggests that there is a connection between the brain's biomolecular processes and the basic structure of the universe" [17].

In the so-called Bio-Universe Model we propose in this article, a multidimensional vacuum with $\mathrm{n}$ dimensions $(\mathrm{n}$ is the cardinal number of natural numbers) is the primordial structure of the universe that governs the evolution of life on planet Earth and in the entire universe. In the Bio-Universe Model, consciousness is mathematically described as the energy of a photon in the $n$-dimensional layer of the vacuum, which has infinite frequency:

$E=n \cdot h \cdot v(7),[18]$. 
where $v$ represents the frequency of the photon, $h$ is Planck's constant and integer $n$ represents the infinite dimension of the vacuum. Consciousness is the vibration of the n-dimensional layer of the vacuum, where the frequency is infinite, and the wavelength value is zero. Out of that, it follows that the velocity $v$ of consciousness is zero:

$v=\infty \cdot 0=0(8)$

Consciousness exists in the entire universe; it is the fundamental property of the universe. Entanglement of EPR type phenomena is transferred via consciousness in the $n$-dimension of the vacuum. The mathematical model of consciousness cannot be considered as a bijective model of consciousness; it only points towards its real nature. Consciousness is subjective and should be searched for subjectively by watching the way the scientific mind functions. The more one develops the ability to observe, to watch one's mind, the more one enters the observer, which is consciousness itself.

Consciousness manifests in the rational part of the human mind as mathematics. The universe is ruled by mathematical laws. The Founder of "Mathematical Universe Hypothesis" (MUH), Max Tegmark, says: Our external physical reality is a mathematical structure [19]. His statement is close to the truth, because we cannot approach matter separately from the multidimensional vacuum in which this matter exists. In higher layers of the vacuum are mathematical structures that govern the universe and also the development of life. In the Bio-Universe Model, an atom is three-dimensional and elementary particles such as protons, electrons, and photons are different vortexes of the four-dimensional layer of the vacuum. What Chinese medicine names "QI energy" (life energy), Indian medicine "Prana", the Bio-Universe Model describes by the higher-dimensional layers of the vacuum [18].

The consciousness we search in two ways. The first way is building scientific models of consciousness, a kind of "mathematization of consciousness" where we try to describe consciousness with mathematics. The other way is experiential conscious research where we experience consciousness. In every human being, consciousness acts as the observer. The conscious observer can see how the human mind works. By regular practicing the observation of thought process we will deepen into consciousness. Rene Descartes said: "Cogito ergo sum"! (I think, so I'm). We go one step further: "I'm aware of the mind, so I'm" [20].

\section{Life, the Universe, and Entropy}

The law of entropy governs matter that consists of atoms and molecules. The law of entropy exists only on the three-dimensional layer of the universe. Protons, electrons, and photons are different vortexes of the multidimensional vacuum. The vacuum energy has no entropy and because of this, protons, electrons, and photons have long lifetimes. Seeing life only as atomic and molecular structure is a reductionist view. The core of life is in the multidimensional vacuum.

Seeing a living organism as a machine that obeys the law of entropy is not the correct perspective. Living organisms are capable of maintaining lower entropy than their geological environment because they are partly composed of multidimensional vacuum energy.

We do not have a single piece of evidence that universal space has entropy. We are measuring entropy in the universe only on the level of the matter that exists in the vacuum. Matter represents $5 \%$ of the energy of the universe and the other $95 \%$ is represented by the energy of a multidimensional vacuum [3]. Only $5 \%$ of the energy in the universe obeys the law of entropy and $95 \%$ does not. In black holes, matter disintegrates back into the energy of the vacuum. In interstellar space, protons are continuously formed out of the vacuum. In cosmology, we are overestimating the importance of entropy. In the universe, $95 \%$ of the energy is in the primordial form of the vacuum energy, which is non-entropic.

At the moment of death, the relationship between the higher-dimensional layers of the multidimensional vacuum, which are non-entropic, and the cells of the organism, which are entropic, is broken. Because the bridge 
between non-entropic layers of energy and cells is broken, a dead body increases its entropy in the process of deterioration.

Prigogine has mathematically described the living organism's property to maintain lower entropy than the geological environment with the model of dissipative structures [21]. We call this property "negentropy". However, the negentropy of living organisms has a physical origin in the higher-dimensional vacuum structures.

Prigogine's idea was that all processes in the universe are irreversible. This is correct. His argument was that irreversible processes in living organisms are different from the irreversible processes in the inorganic part of the universe because they produce negentropy. This is also correct. What he missed is that the physical origin of self-organization capacities of living organisms and their potential of self-repair are in the multidimensional vacuum. In today's science, living organisms must be considered as the interconnectedness of the multidimensional vacuum and their atomic and molecular structure. This view is the basis for integrative medicine, which sees and cures disease on all levels of life.

Black holes as the rejuvenating systems of the universe and are keeping entropy of the universe as a whole constant. Increasing of the entropy of matter in the universe is temporal and does not increase the total entropy. The matter has inherent property all over the universe to develop into the systems which tend to the constant entropy of the universe. That's why the evolution of life is a universal process running in the entire universe. Self-organization of matter which is the negentropy process is possible because life is evolving in the universe in which entropy is constant.

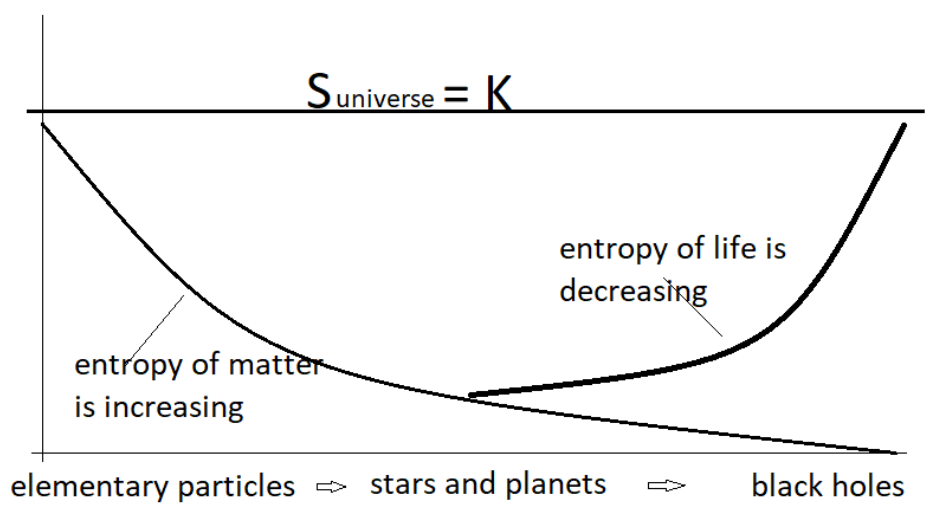

Figure 2: Negentropy of life in the universe with constant entropy

\section{Beyond the geocentric paradigm of life}

The universe is a system with several subsystems. From our perspective, the Milky Way galaxy is the first subsystem of the universe; the solar system is the second subsystem; planet Earth is the fourth subsystem and life on the planet is the fifth subsystem. Biology of the past century has approached the evolution of life as something that has occurred on the Earth's surface. This geo-centric approach in biology is a flawed approach that does not consider the universal system and all subsystems in which the Earth exists. The Bio-Universe Model approaches life as a phenomenon occurring in the multidimensional vacuum, which is the fundamental arena of the universe. The vacuum, which is the physical origin of universal space, is the fundamental physical circumstance in which life has developed and is still developing. 20th-century biology has seen life as a complex bio-molecular system with the property of self-organization. The Bio-Universe Model suggests that selforganization is deeply related to the physical space in which life as a complex bio-molecular system exists.

Experimental data presented in this article confirms in living matter that the vacuum is more involved than in ordinary non-living matter. This is valid also for gravitational fluctuations that are stronger in living mass than in the same dead mass, which results in the weight of a living organism being slightly larger (in worms by about 
one million part) than the weight of the same dead organism. This experiment proves that life is intrinsically related to a dynamic vacuum, which is a fundamental physical property of the universe.

Further, more than 50 stable organic molecules have been detected in the interstellar medium [22]. There are many planets with similar characteristics to our planet: "There is a large parameter space of possible physical characteristics of Earth-like extrasolar planets, and a more careful study of time variation and surface features is recommended" [23]. Thinking that life has evolved only on planet Earth has become unscientific. We have enough experimental data for a paradigm shift that reaches beyond geo-centrism. Considering the vacuum as the fundamental arena of the universe, we can assume that the vacuum represents the fundamental physical parameter that determines the development of life. With this view, we are surpassing the geocentric view of the evolution of life, which is now seen as a larger universal process running in the entire universe. This article is a humble attempt to relate life to the multidimensional vacuum. To connect biology and cosmology.

\section{Conclusions}

The universe is a non-created system in a permanent dynamic equilibrium. The idea of the beginning of the universe with the Big- Bang is a relic of 20th-century cosmology and does not fit today's scientific thought. The evolution of life on our planet is a consistent part of the universal dynamics that run the entire universe and is deeply related to the non-entropic properties of universal space.

\section{References:}

1. NASA https://wmap.gsfc.nasa.gov/universe/uni_shape.html (2014)

2. K. A. Bronnikov, R. I. Budaev, A. V. Grobov, A. E. Dmitriev, Sergey G. Rubin, Inhomogeneous compact extra dimensions, https://arxiv.org/abs/1707.00302 (2017)

3. Amrit Šorli Srečko, Mass-Energy Equivalence Extension onto a Superfluid Quantum Vacuum, Scientific Reports, 9, Article number: 11737 (2019) https://doi.org/10.1038/s41598-019-48018-2

4. Amrit Šorli Srečko, Black Holes are rejuvenating Systems of the Universe, Journal of Advances of Physics, Vol 17 (2020) DOI: https://doi.org/10.24297/jap.v17i.8620

5. Fiscaletti, D., Sorli, A.: Perspectives of the numerical order of material changes in timeless approaches in physics. Found. Phys. 45(2), 105-133 (2015) https://doi.org/10.1007/s10701-014-9840-y

6. Fiscaletti, D. \& Sorli, A. Quantum Stud.: Math. Found. Searching for an adequate relation between time and entanglement,(2017) 4: 357. https://doi.org/10.1007/s40509-017-0110-5

7. Zwicky, F. On the Red Shift of Spectral Lines through Interstellar Space. PNAS 15:773-779. (1929) $10.1073 /$ pnas.15.10.773

8. Lawrence H. Dam, Asta Heinesen, David L. Wiltshire, Apparent cosmic acceleration from Type Ia supernovae, Monthly Notices of the Royal Astronomical Society, Volume 472, Issue 1 ( 2017) 10.1093/mnras/stx1858

9. Amrit Šorli Srečko, The End of Big Bang Cosmology - Bijective Physics, Amazon (2020)

10. Kormendy, John; Ho, Luis (2013). "Coevolution (Or Not) of Supermassive Black Holes and Host Galaxies". Annual Review of Astronomy and Astrophysics. 51 (1): 511-653. arXiv:1304.7762

11. Emilio Del Giudice, Antonella De Ninno, Martin Fleischmann, Giuliano Mengoli, Marziale Milani, Getullio Talpo \& Giuseppe Vitiello (2005) Coherent Quantum Electrodynamics in Living Matter, Electromagnetic Biology and Medicine, 24:3, 199-210, DOI: 10.1080/15368370500379574 
12. Grandpierre A. Biologically Organized Quantum Vacuum and the Cosmic Origin of Cellular Life. In: Tymieniecka AT. (eds) Phenomenology of Space and Time. Analecta Husserliana (The Yearbook of Phenomenological Research), vol 116. Springer, Cham (2014) https://doi.org/10.1007/978-3-319-02015$\underline{010}$

13. Igor Jerman. The Origin of Life from Quantum Vacuum, Water and Polar Molecules. American Journal of Modern Physics. Special Issue: Insufficiency of Big Bang Cosmology. Vol. 5, No. 4-1, 2016, pp. 34-43. doi: 10.11648/j.ajmp.s.2016050401.16

14. Slawinski J., Photon Emission from Perturbed and Dying Organisms: Biomedical Perspectives, Forsch Komplementärmed Klass Naturheilkd 2005;12:90-95 https://www.ncbi.nlm.nih.gov/pubmed/15947467

15. Sorli A., Patro S., Fiscaletti D., Unified Field Theory Based on Bijective Methodology, NeuroQuantology, Vol 16, Num 11, pp: 55-67 (2018) 10.14704/nq.2018.16.11.1855

16. Soul has Weight, Physician Thinks". The New York Times. 11 March 1907. Archived from the original on 17 July 2017. Retrieved 16 July 2017.

17. Stuart Hameroff, Roger Penrose, Consciousness in the universe A review of the 'Orch OR' theory, Physics of Life Reviews 11 (2014) 39-78 https://doi.org/10.1016/j.plrev.2013.08.002

18. Sorli A., Dobnikar U., Fiscaletti D., Koroli V., Advanced Relativity: Multidimensionality of Consciousness and Mind, Origin of Life, PSI Phenomena, NeuroQuantology 2017; 15(2) 10.14704/nq.2017.15.2.1020

19. Tegmark M., The Mathematical Universe, Foundations of Physics. 38 (2): 101-150. (2008). https://doi.org/10.1007/s10701-007-9186-9

20. Sorli A., Kaufman S., Experiential Consciousness Research, NeuroQuantology 2018; 16(3) 10.14704/nq.2018.16.3.1132

21. Nicolis G., Prigogine I., Self-Organization in Nonequilibrium Systems: From Dissipative Structures to Order through Fluctuations, John Wiley, New York, (1977).

22. Michel Nuevo, Jan Hendrik Bredehöft, Uwe J. Meierhenrich, Louis d'Hendecourt, and Wolfram H.-P. Thiemann, Urea, Glycolic Acid, and Glycerol in an Organic Residue Produced by Ultraviolet Irradiation of Interstellar/Pre-Cometary Ice Analogs, Astrobiology 2010 10:2, 245256 https://doi.org/10.1089/ast.2009.0358

23. David J. Des Marais, Martin O. Harwit, Kenneth W. Jucks, James F. Kasting, Douglas N.C. Lin, Jonathan I. Lunine, Jean Schneider, Sara Seager, Wesley A. Traub, and Neville J. Woolf, Remote Sensing of Planetary Properties and Biosignatures on Extrasolar Terrestrial Planets, Astrobiology 2002 2:2, 153181 https://doi.org/10.1089/15311070260192246 